\title{
Estudo da mortalidade materna no Nordeste Brasileiro, de 2009 a 2018
}

\author{
Study of Maternal Mortality in Northeast Brazil, from 2009 to 2018
}

Estudio de Mortalidad Materna en el Noreste de Brasil, de 2009 a 2018

Lucicleide Oliveira Santos ${ }^{1 *}$, Vívian Francielle de Freitas Nascimento ${ }^{1}$, Fernanda de Lourdes da Costa Oliveira Rocha ${ }^{1}$, Ellen Tayanne Carla da Silva ${ }^{1}$.

\section{RESUMO}

Objetivo: Esta pesquisa objetivou analisar o perfil epidemiológico dos óbitos maternos ocorridos no Nordeste brasileiro entre 2009 e 2018. Métodos: Trata-se de um estudo retrospectivo, descritivo, dos óbitos maternos, ocorridos entre 2009 e 2018 no Nordeste, obtidos por meio do Departamento de Informática do Sistema Único de Saúde. Resultados: No Nordeste, ocorreram 5.675 óbitos maternos no intervalo de estudo o que fez com que a região apresentasse razão de mortalidade materna de 68,04 óbitos a cada 100 mil nascidos vivos, comparando o ano de 2009 com o ano de 2018 , houve uma redução de $1,74 \%$ no número absoluto de mortes maternas. Outras doenças da mãe que complicam a gravidez, parto e puerpério ocupou o primeiro lugar entre as principais causas de morte materna, com percentual de $32,24 \%$. Quanto às características sociodemográficas, mulheres na faixa etária de 20 a 29 anos, de cor parda, solteiras e possuindo escolaridade entre 8 e 11 anos, foram as que mais morreram. Conclusão: Conclui-se que, embora a região Nordeste tenha apresentado uma redução no Coeficiente de Mortes Maternas, intervenções na área da saúde, como aumento da cobertura de pré-natal e melhorias na assistência as mulheres nordestinas são de extrema necessidade.

Palavras-chave: Determinantes sociais de saúde, Morte materna, Saúde pública.

\begin{abstract}
Objective: This research aims to analyze the epidemiological profile of maternal deaths that occurred in Northeast Brazil between 2009 and 2018. Methods: It is a retrospective and descriptive study of maternal deaths, which occurred in the States of Northeast Brazil, between 2009 and 2018. The amount of deaths was collected from the Department of Informatics of the Unique Health System. Results: In Northeast Brazil, occurred 5.675 maternal deaths during the study interval, which made this region has a maternal mortality rate of 68,04 deaths per 100 thousand live births, comparing the year 2019 to 2018, The Northeast region had a reduction of $1,75 \%$ in the total amount of maternal deaths. Other diseases that affect pregnancy, childbirth and the puerperium occupied the first place among the main causes of maternal death, with a percentage of $32,24 \%$. As for sociodemographic characteristics, it was observed that women in the age group of 20 to 29 years old, brown-skinned, single and having schooling between 8 and 11 years old were the ones who died the most. Conclusion: It is concluded that, although the Northeast region has presented a reduction in the Maternal Mortality Coefficient, interventions in the health area, such as increased prenatal coverage and improvements in assistance to Northeastern women are extremely necessary.
\end{abstract}

Keywords: Social determinants of health, Maternal death, Public health.

1 Universidade Mauricio de Nassau (UNINASSAU), Caruaru - PE.

*E-mail: cleyde_14@hotmail.com 


\section{RESUMEN}

Objetivo: Esta investigación tuvo como objetivo analizar el perfil epidemiológico de las muertes maternas ocurridas en el noreste de Brasil entre 2009 y 2018. Métodos: se trata de un estudio descriptivo retrospectivo de las muertes maternas ocurridas entre 2009 y 2018 en los estados del noreste de Brasil. obtenido a través del Departamento de Informática del Sistema Único de Salud. Resultados: En Noroeste de Brasil se registraron 5.675 muertes maternas en el intervalo del estudio, lo que provocó que la región presentara una razón de mortalidad materna de 68.04 muertes por cada 100 mil nacidos vivos, comparando el año 2009 con el año 2018, el Nordeste mostró una reducción del 1,74\% en el número absoluto de muertes maternas. Otras enfermedades maternales que complican el embarazo, el parto y el puerperio ocuparon el primer lugar entre las principales causas de muerte materna, con un porcentaje del $32,24 \%$. En cuanto a las características sociodemográficas, se observó que las mujeres en el grupo de edad de 20 a 29 años, de color marrón, solteras y con escolaridad entre 8 y 11 años, fueron las que más fallecieron. Conclusión: Se concluye, aunque la región Nordeste ha presentado una reducción en el Coeficiente de Muertes Maternas, las intervenciones en el área de salud, como un aumento de la cobertura prenatal y mejoras en la atención a las mujeres nororientales, son sumamente necesarias.

Palabras clave: Determinantes sociales de la salud, Muerte materna, Salud pública.

\section{INTRODUÇÃO}

A morte materna é aquela ocorrida durante a gestação ou no puerpério, até 42 dias, e expressa a fragilidade no acesso adequado aos serviços de saúde e as condições socioeconômicas desfavoráveis, permanece um problema de saúde pública, sendo possível relacionar os índices de mortalidade materna às condições de desenvolvimento humano e socioeconômico (LOPES FBT, et al., 2017; LIMA MRG, et al., 2017; GOMES JO, et al., 2018).

A assistência inadequada às mulheres na atenção básica durante o período do pré-natal associada à má gerência dos recursos financeiros são alguns dos fatores de risco para a ocorrência de mortes maternas, também elevam a vulnerabilidade deste público as condições de trabalho, a baixa situação econômica, e a distância entre a residência e o serviço de saúde (SILVA JVCP, et al., 2020; CORTINHAS ABB, et al., 2019; ESTIMA NM e ALVES SV, 2019).

Taxas elevadas de mortalidade materna compõem um quadro de violação dos direitos humanos de mulheres e de crianças. Atendimentos inadequados e carentes de humanização são consequência da falta de atualização dos profissionais, que por estarem despreparados contribuem para a ocorrência de mortes maternas por causa obstétrica direta (SILVA JVCP, et al., 2020; CORTINHAS ABB, et al., 2019).

De acordo com a definição da Classificação Internacional de Doenças (CID-10), as causas de mortalidade materna podem ser classificadas em: causas obstétricas diretas (as que são consequências de complicações da gravidez, parto ou puerpério, ou resultam de tratamentos incorretos) e causas obstétricas indiretas (que são consequências do agravamento de doenças prévias da mãe). É de suma importância destacar que são passíveis de prevenção quase todas as causas diretas. No Brasil, $66,7 \%$ das mortes maternas são resultantes de causas obstétricas diretas (DIAS JMG, et al., 2015).

As síndromes hipertensivas específicas gestacionais (SHEG) e as síndromes hemorrágicas (SH) são as principais causas de morte materna por causa obstétrica direta, seguidas por trombose, infecções puerperais, ruptura prematura das membranas, descolamento prematuro da placenta, atonia uterina, entre outras. Estima-se que em média 830 mulheres chegam a óbito todos os dias durante a gravidez ou parto, configurando um valor extremamente elevado de acordo com a Organização Mundial de Saúde. Estudos apontaram que entre as causas diretas de óbito materno, a hemorragia pós- parto, caracterizada pela perda sanguínea acima de $500 \mathrm{ml}$ após parto vaginal e mais de $1000 \mathrm{ml}$ após cirurgia cesariana, no período de $24 \mathrm{~h}$ imediatas, e a eclampsia, foram predominantes, sendo consideradas como emergências obstétricas e as principais causas de morbimortalidade materna (LOPES FBT, et al., 2017; AVELAR RAA, 2019; SZWARCWALD CL, et al., 2014; LOMBARDO M e ESERIAN JK, 2016; SILVA BL e ALMEIDA MG, 2019). 
A qualidade da assistência oferecida durante o período gravídico-puerperal com respeito aos direitos de cidadania das mulheres, captação precoce da gestante no pré-natal, encaminhamento ao pré-natal de alto risco e valorização das queixas apresentadas pelas gestantes constituem ferramentas de grande importância na prevenção de complicações clínicas e obstétricas, o que contribui diretamente para evitar a ocorrência de mortes maternas, em especial as de causa obstétrica direta (DIAS EG, et al., 2018; RUAS CAM, et al., 2020).

Consultas de pré-natal realizadas com qualidade refletem diretamente nas taxas de mortalidade materna, pois é durante essas consultas que as gestantes são acolhidas e conduzidas por meio da assistência de uma equipe multiprofissional de saúde, composta entre outros profissionais, pelo enfermeiro, que ao prestar orientações as gestantes desempenham uma função de fundamental importância, e por esse motivo devem ser qualificados e atualizados para atender as necessidades da mulher durante o período gestacional e o puerpério (SILVA JVCP, et al., 2020; DIAS EG, et al., 2018).

Sendo assim, conhecer as principais causas que favorecem a ocorrência da mortalidade materna notificadas nos sistemas de informação, é indispensável para auxiliar no planejamento e na construção de políticas eficazes de assistência às gestantes, parturientes e puérperas de modo a intervir precocemente nas causas geradoras de morbimortalidade de mulheres durante o período grávido-puerperal (LIMA MRG, et al., 2017).

Neste sentido, este trabalho tem como objetivo analisar o perfil epidemiológico dos óbitos maternos ocorridos no Nordeste brasileiro entre 2009 e 2018.

\section{MÉTODOS}

Trata-se de um estudo retrospectivo, descritivo, dos óbitos maternos, ocorridos entre 2009 e 2018 nos Estados do Nordeste brasileiro. Foram extraídos junto ao Sistema de Informação sobre Mortalidade (SIM), e Sistema de Informação sobre Nascidos Vivos (SINASC) do Departamento de Informática do Sistema Único de Saúde (DATASUS) do Ministério da Saúde (MS) os dados necessários à construção da presente pesquisa. Por se tratar de pesquisa com base de dados secundários e de domínio público não houve a necessidade de aprovação por comitê de ética, visto que não há riscos à população por identificação nominal.

A Região de estudo corresponde ao Nordeste Brasileiro, composto por nove Estados, sendo eles: Alagoas, Bahia, Ceará, Maranhão, Paraíba, Piauí, Pernambuco, Rio Grande do Norte e Sergipe. De acordo com dados do Instituto Brasileiro de Geografia e Estatística, no ano de 2019 o Nordeste apresentou uma população de 53.081.950 habitantes, uma área de 1.562.387,725 Km², e uma Densidade Demográfica de $30,54 \mathrm{hab} . / \mathrm{km}^{2}$.Ocupando, portanto, $18,3 \%$ do território nacional.

Com base na classificação do CID-10 foram estudados os óbitos maternos por causa direta, indireta e por Morte Materna Não Especificada, cuja causa básica foi um dos seguintes códigos da CID-10: O14Hipertensão com proteinúria significativa, O15- Eclampsia, O45- Descolamento Prematuro da Placenta, O62- Anormalidades de Contração Uterina, O72- Hemorragia Pós-Parto, O75- Outras Complicações do Trabalho de Parto e do Parto NCOP, O85- Infecção Puerperal, 088- Embolia de Origem Obstétrica, O90Complicação do Puerpério NCOP, O95- Morte Obstétrica de causa NE, O99- Outras Doenças Maternas COP complicadas na Gravidez, Parto e Puerpério.

Por meio das informações obtidas através da consulta aos bancos de dados do SIM e do SINASC disponibilizadas no site do DATASUS/MS foi possível realizar o cálculo do Coeficiente de Mortalidade Materna, segundo características sociodemográficas e do percentual das causas acima descritas para 0 período de 2009 a 2018. O coeficiente de Mortalidade Materna foi calculado a partir de:

\section{"Número de óbitos maternos" / "Número de Nascidos vivos." "x 100.000"}

As variáveis utilizadas junto ao SIM/DATASUS/MS referentes às características sociodemográficas foram as seguintes: ano do óbito, região, unidade da federação, idade da mãe, escolaridade, cor/raça. Após obtenção dos dados estes foram organizados utilizando a ferramenta Microsoft Excel $2010 \AA$, pertencente ao pacote Microsoft Office $2010 \AA$ for Windows $\AA$, de maneira a possibilitar o cálculo do percentual das categorias instituídas, sendo os resultados representados por tabelas e figuras. 


\section{RESULTADOS}

No Nordeste Brasileiro, ocorreram 5.675 óbitos maternos no intervalo de 10 anos (2009-2018). Este valor fez com que a região apresentasse coeficiente de mortalidade materna de 68,04 óbitos a cada 100 mil nascidos vivos nesse período. Comparando o ano de $2009 \mathrm{com}$ o ano de 2018, o Nordeste apresentou uma redução de 1,74\% no número absoluto de mortes maternas. Esta redução foi diferente para cada Estado, tendo a Bahia apresentado redução de 5,08\%, Sergipe, 6,58\%, Pernambuco, 1,23\%, Maranhão, 1,07\% e o Piauí, $0,49 \%$ no número absoluto de mortes maternas. Em contrapartida, os outros Estados do Nordeste apresentaram um aumento em relação ao absoluto de mortes, Alagoas, 2,87\%, Ceará, 0,36\%, Rio Grande do Norte, $0,35 \%$ e Paraíba, 0,3\%.

Constatou-se que o Estado do Maranhão foi o que apresentou os maiores índices nesses anos de estudo, com um coeficiente médio de 87,73, apresentando uma média maior que a Regional. Em 2018, o Estado do Piauí apresentou um coeficiente de mortalidade materna de 82,84 óbitos maternos a cada 100 mil nascidos vivos, valor que supera os demais Estados da Região: Bahia, 56,98, Sergipe, 58,38, Alagoas, 49,53, Pernambuco, 54,95, Paraíba, 53,15, Rio Grande do Norte, 51,97, Ceará, 67,68 e Maranhão, 78,53, ficando em segundo lugar (Tabela 1).

Tabela 1 - Coeficiente de Mortalidade Materna segundo os Estados do Nordeste, 2009 a 2018.

\begin{tabular}{ccccccccccc}
\hline Ano & MA & PI & CE & RN & PB & PE & AL & SE & BA & Nordeste \\
\hline $\mathbf{2 0 0 9}$ & 83,31 & 84,32 & 65,45 & 49,05 & 51,65 & 60,64 & 32,45 & 87,72 & 87,72 & 71,32 \\
$\mathbf{2 0 1 0}$ & 101,20 & 93,07 & 70,64 & 31,47 & 47,70 & 54,18 & 55,39 & 73,99 & 73,99 & 69,55 \\
$\mathbf{2 0 1 1}$ & 85,87 & 85,75 & 67,66 & 68,61 & 51,07 & 54,97 & 47,90 & 67,90 & 67,90 & 67,57 \\
$\mathbf{2 0 1 2}$ & 71,53 & 100,08 & 67,00 & 65,97 & 47,56 & 48,10 & 45,71 & 70,00 & 70,00 & 64,25 \\
$\mathbf{2 0 1 3}$ & 93,91 & 90,48 & 74,47 & 47,01 & 70,36 & 63,63 & 59,06 & 78,19 & 78,19 & 74,01 \\
$\mathbf{2 0 1 4}$ & 79,44 & 68,83 & 65,28 & 74,83 & 53,88 & 61,33 & 102,20 & 71,56 & 71,56 & 70,46 \\
$\mathbf{2 0 1 5}$ & 99,52 & 83,24 & 49,05 & 61,10 & 64,31 & 67,58 & 57,41 & 68,71 & 68,71 & 68,41 \\
$\mathbf{2 0 1 6}$ & 99,55 & 80,88 & 58,62 & 68,33 & 87,37 & 54,31 & 51,91 & 60,05 & 60,05 & 67,33 \\
$\mathbf{2 0 1 7}$ & 84,97 & 72,09 & 65,73 & 75,72 & 62,62 & 61,80 & 31,77 & 64,68 & 64,68 & 65,46 \\
$\mathbf{2 0 1 8}$ & 78,53 & 82,85 & 67,69 & 51,97 & 53,15 & 54,95 & 49,53 & 56,98 & 56,98 & 61,90 \\
\hline
\end{tabular}

Fonte: Santos LO, et al., 2020.

As principais causas de óbitos no Nordeste Brasileiro, nos anos de 2009 a 2018, foram: outras doenças da mãe que complicam a gravidez, parto e puerpério (32,24\%); eclampsia (15,96\%); hipertensão gestacional com proteinúria significativa $(9,92 \%)$; hemorragia pós-parto $(7,73 \%)$; Embolia de origem obstétrica $(6,29 \%)$, infecção puerperal $(6,17 \%)$, anormalidades da contração uterina $(6,07 \%)$, complicações do puerpério não classificada em outra parte, $(4,46 \%)$; descolamento prematuro da placenta (3,80\%); outras complicações do trabalho de parto e do parto não classificado em outra parte $(3,73 \%)$; morte obstétrica de causa não especificada, (63\%).

Diferenças nas causas de morte materna foram observadas entre os Estados da região Nordeste, na Bahia a principal causa foi devido a outras doenças maternas que complicam a gravidez, o parto e 0 puerpério (38,51\%); assim como nos demais Estados do Nordeste (PI 33,11\%, CE 31,52\%, RN 31,61\%, PB $24,90 \%$, PE 40,03\%, AL 29,28\% e SE 32,50), excetuando o Estado do Maranhão onde a principal causa de morte foi por eclampsia (24,36\%); No Piauí, Ceará, Pernambuco, Alagoas, Sergipe e Bahia a segunda principal causa de morte também foi por eclampsia, 19,34\%, 16,48\%, 12,36\%, 14,36\%, 20\% e $11,58 \%$ respectivamente; no Rio Grande do Norte a hemorragia pós-parto (12,95\%) e a eclampsia (12,95\%) dividem a segunda colocação entre as principais causas de morte; portanto na Região Nordeste as doenças maternas que complicam a gravidez, o parto e o puerpério e a eclampsia ocupam a primeira e segunda colocação entre as onze principais causas de morte materna, $32,24 \%$ e $15,96 \%$, respectivamente, como se observa na (Tabela 2). 
Tabela 2 - Total parcial abaixo das 11 principais causas de morte materna no Nordeste de acordo com o CID-10.

\begin{tabular}{|c|c|c|c|c|c|c|c|c|c|c|}
\hline $\begin{array}{c}\text { Categoria CID- } \\
10\end{array}$ & MA & PI & CE & RN & PB & PE & $A L$ & SE & BA & Nordeste \\
\hline $\begin{array}{c}014 \\
\text { Hipertensão } \\
\text { gestacional } \\
\text { c/proteinuria } \\
\text { signif }\end{array}$ & $8,84 \%$ & $8,85 \%$ & $9,92 \%$ & $9,33 \%$ & $14,86 \%$ & $10,05 \%$ & $4,42 \%$ & $17,50 \%$ & $9,65 \%$ & $9,92 \%$ \\
\hline $\begin{array}{c}015 \\
\text { Eclampsia }\end{array}$ & $24,36 \%$ & $19,34 \%$ & $16,48 \%$ & $12,95 \%$ & $13,25 \%$ & $12,36 \%$ & $14,36 \%$ & $20,00 \%$ & $11,58 \%$ & $15,96 \%$ \\
\hline $\begin{array}{c}045 \\
\text { Descolamento } \\
\text { prematuro da } \\
\text { placenta }\end{array}$ & $7,36 \%$ & $3,61 \%$ & $2,40 \%$ & $2,59 \%$ & $1,61 \%$ & $1,98 \%$ & $3,31 \%$ & $3,75 \%$ & $4,05 \%$ & $3,80 \%$ \\
\hline $\begin{array}{c}062 \\
\text { Anormalidades } \\
\text { da contracao } \\
\text { uterina }\end{array}$ & $7,23 \%$ & $5,25 \%$ & $5,60 \%$ & $3,63 \%$ & $5,22 \%$ & $6,26 \%$ & $7,73 \%$ & $5,63 \%$ & $6,08 \%$ & $6,7 \%$ \\
\hline $\begin{array}{c}072 \\
\text { Hemorragia } \\
\text { pos-parto }\end{array}$ & $7,36 \%$ & $7,21 \%$ & $6,24 \%$ & $12,95 \%$ & $7,23 \%$ & $7,25 \%$ & $6,63 \%$ & $10,00 \%$ & $8,30 \%$ & $7,73 \%$ \\
\hline $\begin{array}{l}\text { O75 Outr } \\
\text { complic do } \\
\text { trab parto e do } \\
\text { parto NCOP }\end{array}$ & $6,16 \%$ & $1,97 \%$ & $4,48 \%$ & $3,63 \%$ & $4,42 \%$ & $1,65 \%$ & $4,97 \%$ & $0 \%$ & $3,47 \%$ & $3,73 \%$ \\
\hline $\begin{array}{c}\text { O85 Infecc } \\
\text { puerperal }\end{array}$ & $7,90 \%$ & $7,54 \%$ & $4,64 \%$ & $9,84 \%$ & $8,84 \%$ & $5,77 \%$ & $10,50 \%$ & $2,50 \%$ & $4,15 \%$ & $6,17 \%$ \\
\hline $\begin{array}{l}\text { O88 Embolia } \\
\text { orig obstetrica }\end{array}$ & $4,02 \%$ & $6,56 \%$ & $7,84 \%$ & $5,70 \%$ & $8,84 \%$ & $8,07 \%$ & $7,18 \%$ & $3,13 \%$ & $5,69 \%$ & $6,29 \%$ \\
\hline $\begin{array}{l}\text { O90 Complic } \\
\text { do puerperio } \\
\text { NCOP }\end{array}$ & $4,15 \%$ & $2,62 \%$ & $7,68 \%$ & $4,15 \%$ & $4,82 \%$ & $3,95 \%$ & $10,50 \%$ & $2,50 \%$ & $2,80 \%$ & $4,46 \%$ \\
\hline $\begin{array}{c}\text { O95 Morte } \\
\text { obstetrica de } \\
\text { causa NE }\end{array}$ & $1,87 \%$ & $3,93 \%$ & $3,20 \%$ & $3,63 \%$ & $6,02 \%$ & $2,64 \%$ & $1,10 \%$ & $2,50 \%$ & $5,69 \%$ & $3,63 \%$ \\
\hline $\begin{array}{l}\text { O99 Outr } \\
\text { doenc mat } \\
\text { COP compl } \\
\text { grav parto } \\
\text { puerp }\end{array}$ & $20,75 \%$ & $33,11 \%$ & $31,52 \%$ & $31,61 \%$ & $24,90 \%$ & $40,03 \%$ & $29,28 \%$ & $32,50 \%$ & $38,51 \%$ & $32,24 \%$ \\
\hline
\end{tabular}

Fonte: Santos LO, et al., 2020.

Com relação à escolaridade materna, observou-se que 1.613 mulheres possuíam de 8 a 11 anos de estudo, o que significa um percentual de $28,42 \%$ do total de óbitos ocorridos no período estudado, seguido de $24,72 \%$ correspondentes aos óbitos de mulheres com escolaridade entre 4 a 7 anos de estudo. Observou-se que do total de óbitos registrados no SIM, 1.248 tinham a escolaridade ignorada, representando um percentual de 21,99\%, conforme demonstrado na (Tabela 3).

Tabela 3 - Percentual de mortes maternas por unidade federativa de acordo com a quantidade de anos de estudo da mãe, de 2009 a 2018.

\begin{tabular}{ccccccccccc}
\hline Escolaridade & MA & PI & CE & RN & PB & PE & AL & SE & BA & Nordeste \\
\hline Nenhuma & $6,91 \%$ & $4,88 \%$ & $3,82 \%$ & $3,90 \%$ & $2,63 \%$ & $5,05 \%$ & $5,73 \%$ & $6,58 \%$ & $3,91 \%$ & $4,79 \%$ \\
\hline 1 a 3 anos & $12,95 \%$ & $13,90 \%$ & $13,60 \%$ & $13,83 \%$ & $9,94 \%$ & $13,30 \%$ & $17,20 \%$ & $12,28 \%$ & $14,34 \%$ & $13,57 \%$ \\
\hline $\mathbf{4}$ a 7 anos & $25,61 \%$ & $26,83 \%$ & $24,94 \%$ & $25,53 \%$ & $17,84 \%$ & $29,80 \%$ & $25,45 \%$ & $27,19 \%$ & $21,48 \%$ & $24,72 \%$ \\
\hline $\mathbf{8}$ a 11 anos & $36,51 \%$ & $22,68 \%$ & $31,50 \%$ & $26,95 \%$ & $17,84 \%$ & $30,54 \%$ & $16,49 \%$ & $36,84 \%$ & $25,12 \%$ & $28,42 \%$ \\
\hline $\begin{array}{c}\text { 12 anos e } \\
\text { mais }\end{array}$ & $58,12 \%$ & $7,07 \%$ & $8,11 \%$ & $8,16 \%$ & $3,22 \%$ & $6,28 \%$ & $3,94 \%$ & $9,21 \%$ & $5,97 \%$ & $6,50 \%$ \\
\hline Ignorado & $11,39 \%$ & $24,63 \%$ & $18,02 \%$ & $21,63 \%$ & $48,54 \%$ & $15,02 \%$ & $31,18 \%$ & $7,89 \%$ & $29,17 \%$ & $22,00 \%$ \\
\hline
\end{tabular}

Fonte: Santos LO, et al., 2020. 
Quanto ao estado civil, a maior prevalência nos registros foi entre as mulheres caracterizadas como solteiras, correspondendo a $48,14 \%$ (n. 2.732) do total de óbitos. Em relação aos Estados no Nordeste, Pernambuco foi o que teve a maior proporção de mortes maternas em mulheres solteiras, com $57,14 \%$ dos casos. Quanto ao estado civil casada, o Nordeste apresentou 25,13\% dos óbitos maternos no período do estudo. O Estado com maior proporção de casos envolvendo mulheres casadas foi o Ceará, com 31,38\%, como pode ser observado na (Tabela 4).

Tabela 4 - Percentual de Mortes Maternas por Estado Civil e Unidade Federativa, de 2009 a 2018.

\begin{tabular}{ccccccccccc}
\hline Estado Civil & MA & PI & CE & RN & PB & PE & AL & SE & BA & Nordeste \\
\hline Solteiro & $48,69 \%$ & $36,34 \%$ & $49,28 \%$ & $52,13 \%$ & $32,75 \%$ & $57,14 \%$ & $46,95 \%$ & $53,95 \%$ & $47,56 \%$ & $48,14 \%$ \\
\hline Casado & $20,16 \%$ & $25,61 \%$ & $31,38 \%$ & $25,89 \%$ & $26,32 \%$ & $26,48 \%$ & $25,09 \%$ & $26,75 \%$ & $23,47 \%$ & $25,13 \%$ \\
\hline Viúvo & $0,88 \%$ & $0,49 \%$ & $0,95 \%$ & $0,35 \%$ & $0,58 \%$ & $0,49 \%$ & $1,08 \%$ & $0,88 \%$ & $0,21 \%$ & $0,60 \%$ \\
\hline $\begin{array}{c}\text { Separado } \\
\text { judicialmente }\end{array}$ & $0,39 \%$ & $0,24 \%$ & $1,43 \%$ & $1,06 \%$ & $0,58 \%$ & $0,74 \%$ & $0,72 \%$ & $1,32 \%$ & $0,75 \%$ & $0,78 \%$ \\
\hline Outro & $22,98 \%$ & $16,83 \%$ & $8,71 \%$ & $10,28 \%$ & $14,04 \%$ & $8,50 \%$ & $7,53 \%$ & $12,28 \%$ & $10,30 \%$ & $12,74 \%$ \\
\hline Ignorado & $6,91 \%$ & $20,49 \%$ & $8,23 \%$ & $10,28 \%$ & $25,73 \%$ & $6,65 \%$ & $18,64 \%$ & $4,82 \%$ & $17,71 \%$ & $12,62 \%$ \\
\hline
\end{tabular}

Fonte: Santos LO, et al., 2020.

Organizando os dados em ordem decrescente, constata-se que a maior prevalência de óbitos maternos foi na faixa etária de 20 a 29 anos (40,56\%), seguida pela faixa entre 30 a 39 anos (36,25\%). Na faixa entre 10 a 19 anos, considerada faixa etária adolescente pela Organização Mundial da Saúde (OMS), encontrouse um percentual de 15,07\%. Entre as adolescentes, $13,71 \%$ eram de 15 a 19 anos e 1,36\% entre 10 a 14 anos. Contudo, no Estado de Alagoas, o percentual de adolescentes foi de $21,86 \%$, sendo o estado com maior percentual de mortes na faixa etária de 10 a 19 anos. A Bahia foi o único estado a apresentar registro com idade ignorada (Tabela 5).

Tabela 5 - Percentual de Mortes Maternas por Faixa Etária e Unidade Federativa, de 2009 a 2018.

\begin{tabular}{ccccccccccc}
\hline Faixa Etária & MA & PI & CE & RN & PB & PE & AL & SE & BA & Nordeste \\
\hline $\mathbf{1 0}$ a $\mathbf{1 4}$ anos & $2,34 \%$ & $1,71 \%$ & $0,84 \%$ & $0,35 \%$ & $0,88 \%$ & $1,72 \%$ & $1,08 \%$ & $0,44 \%$ & $1,17 \%$ & $1,36 \%$ \\
\hline $\mathbf{1 5}$ a $\mathbf{1 9}$ anos & $18,60 \%$ & $16,10 \%$ & $12,05 \%$ & $11,35 \%$ & $12,87 \%$ & $12,44 \%$ & $20,79 \%$ & $11,40 \%$ & $10,91 \%$ & $13,71 \%$ \\
\hline $\mathbf{2 0}$ a $\mathbf{2 9}$ anos & $44,79 \%$ & $42,68 \%$ & $39,14 \%$ & $43,97 \%$ & $37,43 \%$ & $40,39 \%$ & $37,28 \%$ & $43,42 \%$ & $38,16 \%$ & $40,56 \%$ \\
\hline $\mathbf{3 0}$ a $\mathbf{3 9}$ anos & $28,14 \%$ & $32,93 \%$ & $39,86 \%$ & $34,75 \%$ & $42,69 \%$ & $37,07 \%$ & $34,77 \%$ & $35,53 \%$ & $39,53 \%$ & $36,25 \%$ \\
\hline $\mathbf{4 0}$ a $\mathbf{4 9}$ anos & $6,13 \%$ & $6,59 \%$ & $8,00 \%$ & $9,57 \%$ & $6,14 \%$ & $8,25 \%$ & $6,09 \%$ & $9,21 \%$ & $9,68 \%$ & $7,95 \%$ \\
\hline $\mathbf{5 0}$ a $\mathbf{5 9}$ anos & $0 \%$ & $0 \%$ & $0,12 \%$ & $0 \%$ & $0 \%$ & $0,12 \%$ & $0 \%$ & $0 \%$ & $0,48 \%$ & $0,16 \%$ \\
\hline $\begin{array}{c}\text { Idade } \\
\text { ignorada }\end{array}$ & $0 \%$ & $0 \%$ & $0 \%$ & $0 \%$ & $0 \%$ & $0 \%$ & $0 \%$ & $0 \%$ & $0,07 \%$ & $0,02 \%$ \\
\hline
\end{tabular}

Fonte: Santos LO, et al., 2020.

Na variável cor/raça, observou-se que 3.745 casos foram registrados em mulheres da cor/raça parda, representando 65,99\% dos óbitos maternos no Nordeste. Destaca-se que 351 (6,19\%) dos registros não continham essa informação. Observou-se que em todos os anos a cor /raça parda predominou seguida da cor branca $(16,62 \%)$. Notou-se que no ano de 2015 , a mortalidade materna da raça/cor parda alcançou seu maior percentual que foi de $70,29 \%$, já a cor/raça branca obteve seu maior percentual no ano de 2013 $(18,42 \%)$ (Gráfico 1). 
Gráfico 1 - Morte Materna por cor/raça de 2009 a 2018.

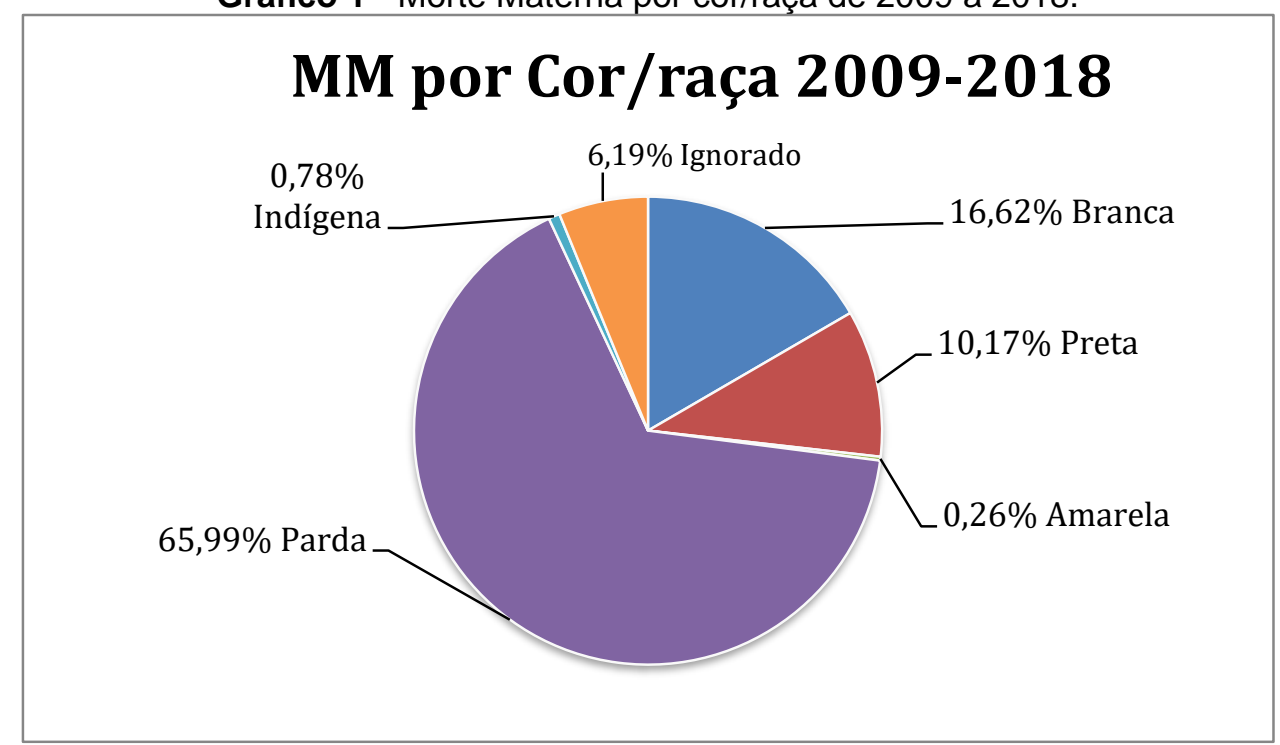

Fonte: Santos LO, et al., 2020.

\section{DISCUSSÃo}

Um excelente indicador da realidade social de um país é o número de mortes maternas, por ser capaz de revelar o nível socioeconômico, a qualidade da assistência, a iniquidade entre gêneros e a eficiência das políticas de saúde pública existentes, relacionando-se inversamente com o grau de desenvolvimento humano (SERQUEIRA JR, et al., 2020).Promovida pelas Nações Unidas, a redução da mortalidade materna fez parte de uma das metas dos Objetivos de Desenvolvimento do Milênio (ODM) e com esse intuito, 180 países, incluindo o Brasil, reuniram esforços. Embora a meta não tenha sido alcançada por todos os países, houve significativos progressos no mundo e cerca de 1.5 milhões de vidas maternas tenham sido poupadas entre 2000 a 2015 (CLARO CP e NASCIMENTO GL, 2018; SOUZA JPA, 2015).

Embora nas duas últimas décadas a mortalidade materna tenha apresentado redução, ainda permanece como um grave problema de saúde pública, e como estratégia nacional foi implantada no Sistema Único de Saúde através da portaria $n^{\circ} 1.459$ de 2011, a Rede Cegonha, que busca garantir ações de humanização ao parto, qualidade na assistência de pré-natal e planejamento familiar, medidas que interferem diretamente na prevenção de óbitos maternos (RIBEIRO RFSA, 2018; MELLO LCF, et al., 2020; GUERRA HS, et al., 2016).

Observa-se que as ações preconizadas pelas políticas de saúde pública, como a Rede Cegonha, influenciam no Coeficiente de mortalidade materna, sendo possível por meio deste estudo verificar que no Nordeste esta taxa oscilou em todo período, no entanto, uma discreta redução no número absoluto de óbitos foi constatado, sendo o ano de 2018 o que apresentou o menor valor, com 61, 90\%, a despeito de 2013, que apresentou 74,01\% (RODRIGUES ARM, CAVALCANTE AES e VIANA BA, 2019).

Os resultados e as informações apresentadas nesta pesquisa são de grande relevância já que dados fornecidos pelos sistemas de informação em saúde contribuem não apenas para a realização de analises epidemiológica, mas também para avaliar a qualidade e execução das políticas públicas, sendo, portanto, ferramentas indispensáveis ao planejamento em saúde (CARVALHO D e MEIRINHO D, 2020).

Estudos apontam que de acordo com fatores sociais, como a idade, a raça, a escolaridade e o estado civil, existe uma população com maior risco para complicações que levem a morte materna, constituindo-se em um grupo mais vulnerável. Esse perfil, também constatado nessa pesquisa, é composto por mulheres na faixa etária adulto-jovem (entre 20-29 anos), com razoável nível de escolaridade (em torno 4 a 7 anos), solteiras e de cor parda (GALVÃO LR, 2019; AZEVEDO LMC, 2018).

A etnia por si só não é um fator de risco, no entanto estudos demonstram que mulheres pardas, assim como as negras, constitui um grupo de maior vulnerabilidade ao óbito materno tal fato pode estar relacionado à predisposição biológica destas mulheres para desenvolver doenças como a hipertensão/pré- 
eclâmpsia. No que diz respeito à inserção social as mulheres negras/pardas se encontram em maior vulnerabilidade, pois a maioria delas encontra-se abaixo da linha da pobreza e com uma taxa de analfabetismo duas vezes maior que as mulheres brancas (MIRANDA BKB, et al., 2019; CARVALHO D e MEIRINHO D, 2020).

Comunidades pobres e com acessibilidade deficitária aos serviços de saúde apresentam maiores índices de mortalidade materna, pois a pobreza econômica das mulheres associada à falta de informações adequadas favorecem a ocorrência de óbitos por causas obstétricas, nesse caso, constata-se que mulheres pobres e desfavorecidas estão mais propensas a morrer prematuramente do que mulheres ricas (SCARTON J, et al., 2020; MENDES A, 2020). Observa-se ainda, que apesar da significativa redução, a mortalidade materna no Brasil e no Nordeste ainda permanece como um grave problema de saúde pública, pois desde 2016 mais de quatro mulheres morrem diariamente em decorrência de complicações do ciclo gravídico-puerperal (ANDRADE JA, 2019).

Foi possível verificar por meio deste estudo que no Nordeste das onze principais causas de morte materna, dez correspondem às causas obstétricas diretas, que são aquelas decorrentes de complicações da gravidez, parto ou puerpério, ou resultam de tratamentos incorretos, neste sentido a qualidade da cobertura de pré-natal interfere diretamente nos fatores de risco para o óbito materno, em contrapartida, o Nordeste ocupa o segundo lugar como a região com menor cobertura de pré-natal (RODRIGUES ARM, CAVALCANTE AES e VIANA BA, 2019; DIAS JMG, 2015; GOIS EC, et al., 2019).

Embora a melhoria na qualidade da assistência ao pré-natal, parto e puerpério impacte diretamente nos índices de mortalidade materna, é necessário que outras estratégias sejam executadas para garantir uma efetiva redução no número de óbitos maternos. Investimentos de cunho social, direcionado a políticas públicas para educação básica, redução da pobreza e das desigualdades sociais se fazem necessários por serem capazes de interferir nas condições de vida e bem-estar das mulheres (LIMA DR, et al., 2016).

É de responsabilidade do Estado à adoção de medidas positivas e protetivas capazes de garantir as mulheres o direito a vida, além de assegurar que os trabalhadores de saúde que as assistem recebam formação adequada, pois, sempre que fatores de ordem médica, social e/ou relativos ao sistema de saúde colocam a vida de uma mulher em risco na gravidez, no parto ou no puerpério é constatada a violação dos seus direitos (JUNIOR RAOF, 2020). Apesar da tendência de queda no período de 2009 a 2018 os resultados apresentados nesse estudo indicam que os valores da razão de mortalidade materna na região são ainda muito preocupantes, e que a busca por estratégias para enfrentar esse desafio se faz necessária.

Um dos grandes obstáculos na mensuração da mortalidade materna decorre do fato de haver falhas no preenchimento das declarações, dando origem a sub-registros, o que dificulta o real monitoramento dos óbitos na região. Sendo este fato uma das fragilidades desse estudo, uma vez que a fonte dos dados é secundária e dependente da qualidade dos dados disponibilizados pelos sistemas de informação do Ministério da Saúde (MARCOMINI EK, et al., 2017; MEDEIROS LT, et al., 2018).

\section{CONCLUSÃo}

Este estudo possibilitou conhecer a situação epidemiológica dos óbitos maternos ocorridos nos Estados do Nordeste Brasileiro e apontou para a necessidade de melhorias na rede assistencial prestada as gestantes, como o aumento na cobertura de pré-natal e a captação precoce de gestantes pelas equipes de atenção básica para inicio imediato do acompanhamento adequado, além da garantia de vinculação destas à maternidade, de maneira a proporcionar a esse publico uma assistência de qualidade e resolutiva em todo ciclo grávido-puerperal, de acordo com as leis e diretrizes que regem o sistema único de saúde. Apesar do declínio na razão de morte materna (RMM), esta permanece como um problema de saúde pública e atinge principalmente grupos em maior vulnerabilidade, além da ocorrência de casos não investigados e da subnotificação, relacionados principalmente aos óbitos maternos por causas obstétricas diretas. Como limitação deste trabalho destaca-se a coleta de dados secundários que por serem provenientes dos sistemas de banco de dados já existentes dependem da qualidade no preenchimento das fichas de notificações e da digitação destas nas bases do SIM e do SINASC não sendo possível garantir a ausência de erros e equívocos. 


\section{REFERÊNCIAS}

1. AVELAR RAA. Atuação da enfermagem na morte materna por hemorragia. Anais dos 17 simpósio de TCC e 14 seminário de IC do Centro Universitário ICESP, 2019; (17): 972-977.

2. AZEVEDO LMC. Mortalidade materna no estado da Paraíba no período de 2007 a 2016. Projeto de pesquisa (Trabalho de Conclusão de Curso). Universidade Federal de Campina Grande - campus Cajazeiras, 2018; $41 \mathrm{p}$.

3. CARVALHO D e MEIRINHO D. O quesito cor/raça: desafios dos indicadores raciais de mortalidade materna como subsídio ao planejamento de politicas públicas em saúde. Reciis - Rev Eletron Comun Inf Inov Saúde, 2020; 14(3):656-80.

4. CLARO CP, NASCIMENTO GL. Óbitos de mulheres em idade fértil observadas no Distrito Federal em 2015. Projeto de pesquisa (Trabalho de conclusão de curso). Faculdade de Ciências da Educação e Saúde, Centro Universitário de Brasília, 2018; 16p.

5. CORTINHAS ABB, et al. Pré-eclâmpsia e mortalidade materna. Revista Caderno de Medicina, 2019, Vol. 2 , № 1.

6. DIAS EG, et al. Ações do Enfermeiro no pré-natal e a importância atribuída pelas gestantes. Revista SUSTINERE, Rio de Janeiro, 2018; 6(1): 52-62.

7. DIAS JMG, et al. Mortalidade materna. Rev. Med. Minas Gerais; $2015 ; 25$ (2): 173-179.

8. ESTIMA NM e ALVES SV. Mortes maternas e de mulheres em idade reprodutiva na população indígena, Pernambuco, 2006-2012. Epidemiol. Serv. Saúde, Brasília, 2019, 28(2): e2018003.

9. GALVÃO LR. Evolução temporal da mortalidade materna em adolescentes e adultas jovens no estado da Bahia no período de 2000-2016. Dissertação (Mestrado em Saúde Coletiva) - Universidade Estadual de Feira de Santana, Feira de Santana, Bahia, Brasil, 2019; 92p.

10. GOIS EC, et al. Mortalidade materna Na Bahia no período de 2012 a 2016. Revista Eletrônica Acervo Saúde / Electronic Journal Collection Health. REAS/EJCH, 2019; Sup(18): e335.

11. GOMES JO, et al. Perfil sociodemográfico e clínico de mortalidade materna. Rev. enferm UFPE online, Recife, 2018; 12(12): 3165-71.

12. GUERRA HS, et al. Análise das ações da rede cegonha no cenário brasileiro. Iniciação Cientifica CESUMAR -2016; 18(1): 73-80.

13. JUNIOR RAOF. Mortalidade materna evitável enquanto injustiça social. Rev. Bras. Saúde Mater. Infant., Recife, 2020, 20(2): 615-622

14. LIMA DR, et al. Análise dos fatores intervenientes da mortalidade materna. Enfermagem Obstétrica, Rio de Janeiro, 2016; 3: e25.

15. LIMA MRG, et al. Alterações maternas e desfecho gravídico-puerperal na ocorrência de óbito materno. Cad. Saúde Colet., Rio de Janeiro, 2017; 25 (3): 324-331.

16. LOMBARDO M, ESERIAN JK. O controle da hemorragia pós-parto e a avaliação da qualidade da ocitocina injetável. Perspectivas médicas, 2016; 27(1): 26-31.

17. LOPES FBT, et al. Mortalidade Materna por Síndromes Hipertensivas e Hemorrágicas em uma Maternidade-escola Referência de Alagoas. Caderno de graduação - ciências biológicas e da saúde, UNIT-AL, novembro, 2017, 4(2): 149-162.

18. MARCOMINI EK, et al. Mortalidade materna em uma regional de saúde no Paraná: análise epidemiológica. Repositório Digital UNICESUMAR, Anais do Encontro Internacional de Produção Científica, Centro Universitário de Maringá, 2017, ISBN978-85-459-0773-2.

19. MEDEIROS LT, et al. Mortalidade materna no estado do Amazonas: estudo epidemiológico. Rev baiana enferm; 2018, 32: e26623.

20. MELLO LCF, et al. Rede Cegonha: percepção de gestantes de alto risco da região do médio vale do Itajaí. Revista Interdisciplinar de Estudo em Saúde da UNIARP. 2020; 9(1): (19)76-87.

21. MENDES A. Perfil epidemiológico da mortalidade materna no hospital de referência nacional da Guiné-Bissau - 2013 a 2018. Dissertação (Mestrado em Saúde Coletiva) - Universidade Federal do Ceará, Fortaleza, 2020, 123p.

22. MIRANDA BKB, et al. Mortalidade Materna: distribuição e causas no estado do Pará entre os anos 2012 a 2016. Revista de Educação, Saúde e Ciência do Xingu. 2019, 1(1).

23. RIBEIRO RFS. A. Redução da mortalidade materna em Pernambuco: realidade ou desafio? Centro de Filosofia e Ciências Humanas; Departamento de Ciência Política, (Programa de Mestrado Profissional em Políticas Públicas). UFPE, Recife, 2018, 77p.

24. RODRIGUES ARM, CAVALCANTE AES, VIANA BA. Mortalidade materna no Brasil entre 2006-2017: análise temporal. RETEP | Revista Tendências da Enfermagem Profissional, 2019; 11(1): 3-9.

25. RUAS CAM, et al. Perfil e distribuição espacial da mortalidade materna. Rev. Bras. Saúde Mater. Infant., Recife, 2020; 20(2): 397-409

26. SERQUEIRA JR, et al. Análise da mortalidade materna por causas relacionadas ao trabalho de parto, parto e puerpério em Goiás no período de 2008 a 2017. Braz. J. of Develop., Curitiba, sep. 2020; 6(9): 68307-68319.

27. SILVA BL, ALMEIDA MG. Mortalidade materna por causa direta: uma revisão integrativa. Projeto de pesquisa (Trabalho de conclusão de curso). Faculdade CESMAC do Sertão, Palmeira dos Índios - AL. 2019. 23p.

28. SILVA JVCP, et al. Fatores de risco e complicações relacionados à mortalidade materna. Ciências Biológicas e de Saúde Unit - Alagoas, outubro, 2020; 6(2): 87-100.

29. SOUZA JP. A mortalidade materna e os novos objetivos de desenvolvimento sustentável (2016-2030). Rev Bras Ginecol Obstet. 2015; 37 (12): 549-51.

30. SCARTON J, et al. Perfil das mortes maternas numa cidade ao sul do Brasil: perspectiva ecossistêmica. Research, Society and Development, 2020, 9(3): e109932524.

31. SZWARCWALD CL, et al. Estimação da razão de mortalidade materna no Brasil, 2008-2011. Cad. Saúde Pública, Rio de Janeiro, 2014; 30 Sup.: S71-S83. 